\title{
CCND3 Gene
}

National Cancer Institute

\section{Source}

National Cancer Institute. CCND3 Gene. NCI Thesaurus. Code C97381.

This gene is involved in cell cycle progression. 